\title{
Novel therapeutic approach for hemophilia using gene delivery of an engineered secreted activated Factor VII
}

\author{
Paris Margaritis, ${ }^{1}$ Valder R. Arruda, ${ }^{1,2}$ Majed Aljamali,1 Rodney M. Camire,1,2 \\ Alexander Schlachterman, ${ }^{1}$ and Katherine A. High ${ }^{1,2,3}$
}

\begin{abstract}
'Division of Hematology, The Children's Hospital of Philadelphia, Abramson Research Center, Philadelphia, Pennsylvania, USA. ${ }^{2}$ Department of Pediatrics, University of Pennsylvania School of Medicine, Philadelphia, Pennsylvania, USA. ${ }^{3}$ Howard Hughes Medical Institute, The Children's Hospital of Philadelphia, Philadelphia, Pennsylvania, USA.
\end{abstract}

\begin{abstract}
Hemophilia is a bleeding disorder caused by mutations in the genes encoding coagulation Factor VIII (FVIII) or FIX. Current treatment is through intravenous infusion of the missing protein. The major complication of treatment is the development of neutralizing Ab's to the clotting factor. Infusion of recombinant activated human Factor VII (rhFVIIa), driving procoagulant reactions independently of human FVIII (hFVIII) or hFIX, has been successful in such patients and could in theory provide hemostasis in all hemophilia patients. However, its high cost and short half-life have limited its use. Here, we report a novel treatment strategy with a recombinant adeno-associated virus vector delivering a modified FVII transgene that can be intracellularly processed and secreted as activated FVII (FVIIa). We show long-term expression, as well as phenotypic correction of hemophilia B mice following gene transfer of the murine FVIIa homolog, with no evidence of thrombotic complications at these doses. These data hold promise for a potential treatment for hemophilia and other bleeding disorders.
\end{abstract}

\section{Introduction}

Hemophilia is an X-linked recessive bleeding disorder caused by mutations in the genes encoding Factor VIII (FVIII; hemophilia A) or FIX (hemophilia B) and is currently treated by repeated intravenous infusions of clotting factor, either prophylactically or in response to bleeding episodes. A major complication in hemophilia treatment is the development of neutralizing Ab's against the infused protein. Clinically referred to as inhibitors (1), they occur in about $3 \%$ and about $20 \%$ of patients with severe hemophilia B and severe hemophilia A, respectively $(2,3)$. Management of acute bleeding episodes in inhibitor patients depends on the inhibitor titer, but the initially available human FVIII-bypassing (hFVIII-bypassing) or hFIX-bypassing agents had a relatively low efficacy $(4,5)$.

More recently, recombinant activated human Factor VII (rhFVIIa) (NovoSeven; Novo Nordisk, Bagsvaerd, Denmark) has been a highly successful alternative treatment for hemophilia inhibitor patients (6). Human FVIIa combines with tissue factor (TF) (Figure 1) to form a complex that catalyzes conversion of FX to activated FX (FXa), thus circumventing either deficiencies of, or inhibitory Ab's to, FVIII or FIX (the FIXa-FVIIIa complex also catalyzes conversion of FX to FXa). rhFVIIa is synthesized as the zymogen form (recombinant FVII, rFVII) and cleaved to the biologically active form (rhFVIIa) during

Nonstandard abbreviations used: activated Factor X (FXa); activated partial thromboplastin time (aPTT); adeno-associated virus (AAV); arginine lysine arginine (RKR); Factor VIII (FVIII); $\gamma$-carboxyglutamic acid (Gla); human activated Factor VII (hFVIIa); human Factor VIII (hFVIII); human $\alpha_{1}$-antitrypsin (hAAT); human embryonic kidney 293 (HEK 293); insulin receptor (INS); kilo international units (KIU); murine activated Factor VII (mFVIIa); murine Factor VII (mFVII); paired amino acid-cleaving enzyme (PACE); plasmid AAV (pAAV); prothrombin time (PT); recombinant AAV (rAAV); recombinant human activated Factor VII (rhFVIIa); thrombin-antithrombin (TAT); tissue factor (TF); vector genomes (vg).

Conflict of interest: The authors have declared that no conflict of interest exists.

Citation for this article: J. Clin. Invest. 113:1025-1031 (2004).

doi:10.1172/JCI200420106. protein purification. It induces hemostasis when administered at supraphysiological doses of $90-110 \mu \mathrm{g} / \mathrm{kg}$ (6). Its most important drawback is its short half-life of 2.7 hours (7), which necessitates frequent infusions and results in high treatment costs, since more than one bolus dose is usually required to treat a bleeding episode successfully, especially if bleeding is severe or if the purpose is to provide hemostasis for surgery (6). One proposed approach has been the continuous infusion of rhFVIIa aimed at maintaining a relatively constant plasma concentration. This approach is less expensive, since a lower total dose can be used (ref. 3, and references therein).

We sought to determine whether a gene transfer approach could circumvent some of the current disadvantages of rhFVIIa protein infusion therapy. As a first step, we engineered a novel hFVII transgene containing a cleavage site for the intracellular protease, paired amino acid-cleaving enzyme (PACE)/furin, and showed that the variant was correctly processed intracellularly and secreted as human activated Factor VII (hFVIIa). We introduced this construct into a recombinant adeno-associated virus (AAV) vector and showed that the vector could direct long-term, high-level transgene expression in normal mice. Because of earlier reports suggesting that hFVIIa does not interact efficiently with murine TF (8-10), we modified a murine FVII (mFVII) construct in an analogous fashion to test efficacy in mice with hemophilia B. In this study, we demonstrate longterm correction of the mouse hemophilia B phenotype following infusion of an AAV vector expressing activated murine FVII (mFVIIa). Moreover, at the doses tested, there was no biochemical or clinical evidence of thrombotic complications at time points up to 6 months after vector injection. This novel approach has the potential to provide hemostasis in all hemophilia patients, whether inhibitors are present or not. Since all patients should be tolerant to activated FVII, the approach avoids altogether the risk of inhibitory $\mathrm{Ab}$ formation. Moreover, the approach may be useful for other inherited bleeding disorders such as Glanzmann's thrombasthenia, 
Bernard-Soulier syndrome, and Factor VII deficiency, as has already been shown for rhFVIIa protein infusion therapy (11-14).

\section{Methods}

Plasmid constructs. The hFVII cDNA (NCBI accession no. AF466933) was cloned in the pcDNA3 vector as a 2.4-kb Bam HI-XbaI fragment (15). Insertion of short amino acid sequences at position Arg152Ile153 was performed using the parent pcDNA3-hFVII vector and either PCR mutagenesis (Stratagene, La Jolla, California, USA) or strand-overlap extension using appropriate primers, with results verified by DNA sequencing. The plasmid constructs were named as follows: hFVII-WT, hFVII-arginine lysine arginine (hFVII-RKR) (forward: 5'-CAAGGCCGAAGGAAGAGGATTGTGGGG-3'; reverse: 5'-CCCCACAATCCTCTTCCTTCGGCCTTG-3'), hFVII-2RKR (forward: $5^{\prime}$ CAAGGCCGAAGGAAGAGGAGGAAGAGGATTGTGGGG-3'; reverse: 5'-CCCCACAATCCTCTTCCTCCTCTTCCT-3'), and hFVIIinsulin receptor (hFVII-INS) (forward: 5'-CCGCGCCCGTCCCGCAAGCGCCGCATTGTGGGGGGCAAGGTGTGCC-3'; reverse: 5' GCGGCGCTTGCGGGACGGTCGGCCTTGGGGTTTGCTGGC-3’). The mFVII-WT was cloned by reverse transcription using mouse liver mRNA (CLONTECH Laboratories Inc., Palo Alto, California, USA) and primers designed from the published sequence (16). The mouse homolog of hFVII-2RKR was generated essentially as described above. To purify the mFVII and FVII-2RKR, a 12-amino acid epitope (thrombin cleavage site of human protein C, EDQVDPRLIDGK, HPC4) was placed at the $\mathrm{C}$ terminus of each of these transgenes by PCR (resulting in plasmids mFVII-HPC4 and mFVII-2RKR-HPC4). The recombinant AAV (rAAV) plasmid was based on plasmid AAV-hAAT-F.IX (pAAVhAAT-F.IX), a vector containing the human $\alpha_{1}$-antitrypsin (hAAT) promoter with four copies of the human ApoE enhancer (17) and a synthetic intron (18).

In vitro expression and adeno-associated virus production. Human embryonic kidney 293 (HEK 293) cells were cultured as previously described (19). Stable cell lines were generated by calcium phosphate transfection (20) and subsequent single-cell clone selection in G418 medium. Clones were assayed for hFVII expression by ELISA (Enzyme Research Laboratories, South Bend, Indiana, USA) or by prothrombin time (PT) for the mFVII-HPC4 and mFVII2RKR-HPC4 transfectants. Helper-free rAAV was produced by triple transfection of HEK 293 cells, as previously described (21). Vector titers were determined by slot-blot hybridization using known DNA standards and a $0.5-\mathrm{kb}$ probe spanning the synthetic intron of the pAAV-hAAT plasmid.

Purification and in vitro analysis of Factor VII proteins. Conditioned medium containing recombinant proteins was supplemented with $10 \mathrm{mM}$ benzamidine (Sigma-Aldrich, St. Louis, Missouri, USA) and stored at $-20^{\circ} \mathrm{C}$. For the human proteins, purification was performed as previously described (19), except that we used a hFVII/VIIa ELISA kit (Diagnostica Stago Inc., Parsippany, New Jersey, USA) according to the manufacturer's instructions. Conditioned medium containing mFVII-HPC4 and mFVII-2RKR-HPC4 was processed as described above and purified using an HPC4 Ab column (provided by Charles and Naomi Esmon, Oklahoma Medical Research Foundation, Oklahoma City, Oklahoma, USA) as previously described $(22,23)$. The protein concentration was calculated using a molecular weight of 50,000 and an extinction coefficient $\left(\mathrm{E}_{280}{ }^{0.1 \%}\right)$ of 1.39 . All proteins were stored at $-80^{\circ} \mathrm{C}$. A rabbit polyclonal $\mathrm{Ab}$ against mFVII and mFVII-2RKR was generated, and the antigen-specific IgG was purified using immunoaffinity chromatography with a mFVII-2RKR-HPC4-coupled column (Green Mountain Antibod- ies Inc. Burlington, Vermont, USA). A part of this Ab was labeled with HRP (Roche, Indianapolis, Indiana, USA) and used with the same unlabeled $\mathrm{Ab}$ in a sandwich ELISA to measure mFVII/FVIIa levels in mouse plasma.

Clotting assays. The PT assay was performed using $50 \mu \mathrm{l}$ of murine plasma sample diluted in buffer (10 mM Tris, $150 \mathrm{mM} \mathrm{NaCl}, 0.01 \%$ BSA) at 1:160, mixed with $50 \mu \mathrm{l}$ of hFVII-deficient plasma (Organon Teknika Corp., West Orange, New Jersey, USA) in a fibrometer (BBL, Cockeysville, Maryland, USA) at $37^{\circ} \mathrm{C}$. Time to clot formation was measured immediately after the addition of $200 \mu \mathrm{l}$ of prewarmed Innovin (recombinant human TF thromboplastin; DADE/Behring, Deerfield, Illinois, USA). The activated partial thromboplastin time (aPTT) assay was performed using $50 \mu$ l of undiluted sample mixed with $50 \mu \mathrm{l}$ of hFIX-deficient plasma (Organon Teknika Corp.) and 50 $\mu \mathrm{l}$ aPTT reagent (Organon Teknika Corp.). Following a 3-minute incubation at $37^{\circ} \mathrm{C}, 50 \mu \mathrm{l}$ of $25 \mathrm{mM} \mathrm{CaCl}_{2}$ were added and the time to clot measured using a fibrometer. Normal range for hemostatically normal C57BL/6 mice in this assay is $27.3-32.4$, average $30.1 \pm 1.88$ seconds, and for hemophilia B mice on a C57BL/ 6 background the range is $52.8-85.3$, average $65.3 \pm 9.86$ seconds. Thrombin-antithrombin (TAT) levels were measured by ELISA on plasma collected by tail transection. The first drop of blood was discarded. The TAT ELISA (Enzygnost TAT, Newark, Delaware, USA) was used according to manufacturer's instructions and has been validated for determination of TAT levels in mice (24).

Animal experiments. All experimental procedures were reviewed and approved by the Institutional Animal Care and Use Committee of The Children's Hospital of Philadelphia. All animal experiments made use of 8- to 12-week-old, hemostatically normal C57BL/6 mice or hemophilia B (25) mice on a C57BL/6 background. rAAV was delivered into the hepatic circulation by injection into the spleen. Blood samples from the tail vein were collected into onetenth volume of $3.8 \%$ sodium citrate, prior to and following AAV delivery at regular time intervals. hFVII/FVIIa levels were monitored by ELISA (Diagnostica Stago Inc.) as previously described (26) or by PT and aPTT (mouse transgenes). Tail clip assays were performed as follows: the tail was placed in $37^{\circ} \mathrm{C}$ normal saline solution for 2

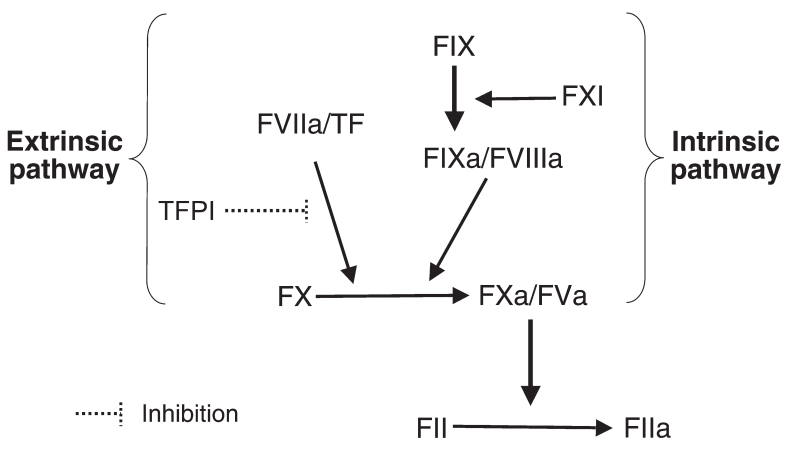

\section{Figure 1}

Role of FVIla in coagulation. Coagulation is initiated through the extrinsic pathway. FVIIa/TF catalyzes conversion of FX to FXa. FXa catalyzes conversion of prothrombin (FII) to thrombin (FIla). Small amounts of thrombin formed through the extrinsic pathway promote coagulation through the intrinsic pathway. Under physiological conditions, the extrinsic pathway is dramatically downregulated by TF pathway inhibitor (TFPI), but the high circulating concentrations of FVIla seen with infusion of the recombinant protein or the AAV-FVII-2RKR vector allow FX activation to occur despite TFPI. 
A
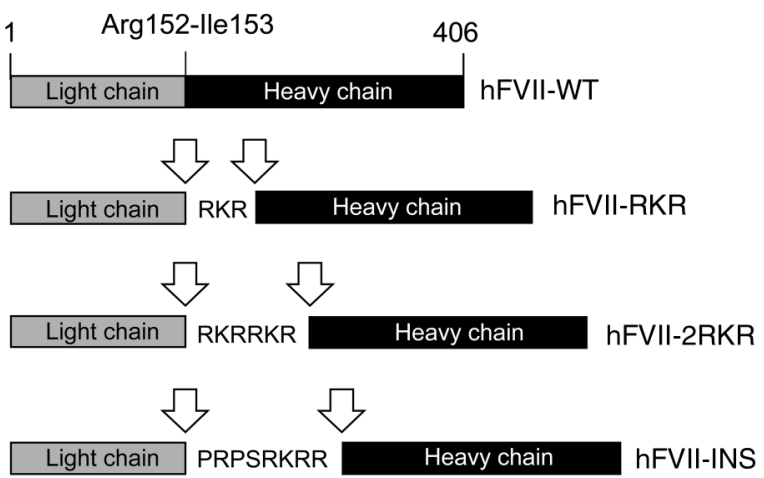

B

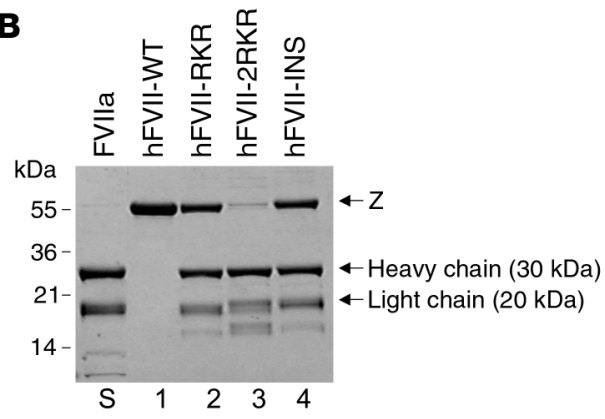

C

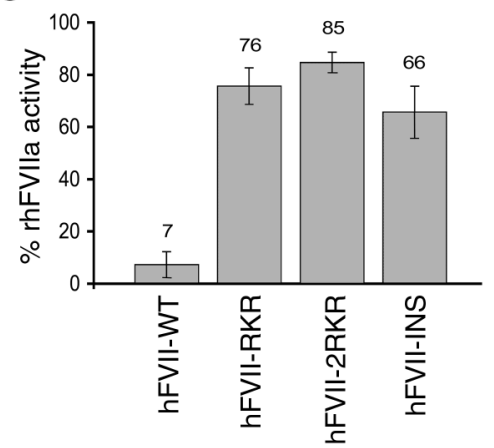

Figure 2

Engineered hFVIla transgenes and in vitro characterization. (A) Schematic representation of the engineered hFVII and hFVIla constructs, showing the light and heavy chains. An open arrow indicates the proposed recognition site of the PACE/furin type intracellular protease. (B) PAGE gel under reducing conditions of $4 \mu \mathrm{g}$ of in vitro-expressed and -purified engineered transgenes: far left lane (labeled S, FVIla) contains rhFVIla as a reference standard; lane 1, hFVII-WT; lane 2, hFVII-RKR; lane 3, hFVII-2RKR; lane 4, hFVII-INS. Arrows point to the following forms: zymogen $(Z)$, heavy chain, and light chain. Molecular weight markers are shown to the left in kilodaltons. (C) In vitro activity based on PT as indicated on top of each bar (mean \%), relative to rhFVIla (100\%): far left, hFVII-WT; center left, hFVII-RKR; center right, hFVII-2RKR; far right, hFVII-INS. Error bars denote mean $\pm 1 \mathrm{SD}$.

minutes and subsequently cut at a 3 -mm diameter. The tail was then immediately placed in $37^{\circ} \mathrm{C}$ normal saline solution and allowed to bleed (or clot) without therapeutic intervention for 15 minutes. The experiment was terminated if necessary at 15 minutes by applying silver nitrate and suturing the transected tail. Blood loss was quantified by measuring the hemoglobin content that accumulated in the saline solution over the 15 -minute time interval before the tail was sutured. Absorbance of the sample was measured at $575 \mathrm{~nm}$, as previously described (27). Data were analyzed using an unpaired Student's $t$ test.

\section{Results}

In vitro characterization of hFVII variant proteins. hFVII, a 406-amino acid, vitamin $\mathrm{K}$-dependent serine protease, circulates predominantly as a zymogen (single-chain precursor molecule), with only a trace amount $(0.1-1 \%)$ in the two-chain, activated form (hFVIIa) (28). To effect a gene transfer approach, we needed a modified transgene with a short amino acid sequence inserted at position 152 (the normal site of cleavage of hFVII that yields hFVIIa) that would be recognized by an intracellular protease of the PACE/furin-type, resulting in a secreted two-chain molecule similar in structure to hFVIIa.

We tested three potential intracellular cleavage sites (Figure 2A). Stable HEK 293 cell lines producing hFVII-WT as well as hFVIIa variants were established, all with roughly similar levels of expression. hFVII-WT was secreted at $1,000 \pm 120 \mathrm{ng} / \mathrm{ml} / 24 \mathrm{~h}$, hFVII-RKR at $1,310 \pm 260 \mathrm{ng} / \mathrm{ml} / 24 \mathrm{~h}, \mathrm{hFVII}-2 \mathrm{RKR}$ at $710 \pm 30 \mathrm{ng} / \mathrm{ml} / 24 \mathrm{~h}$, and
hFVII-INS at $1,080 \pm 130 \mathrm{ng} / \mathrm{ml} / 24 \mathrm{~h}$. Recombinant proteins were purified, and SDS-PAGE analysis indicated that all the engineered hFVII variants were secreted in a predominantly activated, twochain form (Figure 2B, lanes 2-4). Moreover, hFVII-WT was secreted as a single-chain molecule, indicating that no activation occurred following secretion into the conditioned medium (Figure 2B, lane 1); this was also confirmed by Western blot analysis of conditioned medium prior to purification (data not shown). These data indicate that secretion of the two-chain engineered hFVIIa variants was a result of intracellular processing at the site of the inserted sequence. In vitro analysis of the purified proteins using a PT assay showed that all hFVIIa variants exhibited similar activities in the range of $66-85 \%$ relative to rhFVIIa (Figure 2C), in agreement with banddensitometric analysis of the gel in Figure 2B. In contrast, hFVIIWT showed a small, $7 \%$ activity relative to rhFVIIa (Figure $2 \mathrm{C}$ ).

Previous work has shown that zymogen hFVII competes with hFVIIa for binding to TF, so that the ratio of hFVII/FVIIa, as well as the total hFVIIa concentration, can influence whether hemostasis is achieved (29). Although our in vitro results indicate that all hFVIIa variants contain zymogen protein as a fraction of the secreted material, autoactivation in the presence of TF resulted in an almost complete conversion of all purified proteins to the twochain, activated form (data not shown). Moreover, with the exception of hFVII-INS, activation of the proteins in the presence of FXa, the most likely candidate for activation of TF-bound FVII during clotting (30), resulted in a similar conversion to the two-chain, activated form (data not shown). Thus, the zymogen form of each vari- 


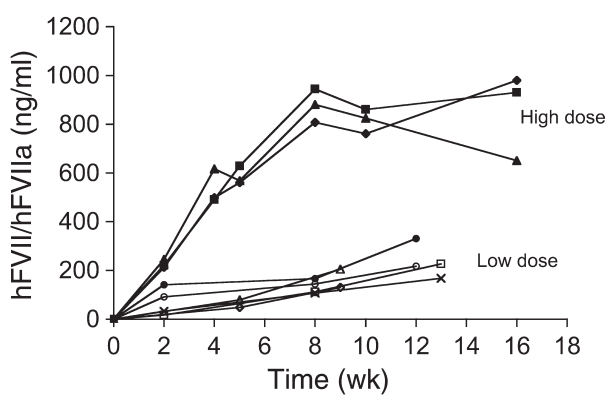

Figure 3

In vivo expression of hFVII-2RKR, following administration of AAV2hAAT-hFVII-2RKR in the hepatic circulation of immunodeficient mice. Two vector dose ranges were used, a low-dose range of $0.7 \times 10^{11}$ to $2 \times 10^{11} \mathrm{vg} /$ mouse and a high-dose range of $0.6 \times 10^{12}$ to $1.2 \times 10^{12}$ $\mathrm{vg} /$ mouse. Each line represents a single animal.

ant (except hFVII-INS) could be fully cleaved to yield activated material in the presence of TF or FXa.

To establish that intracellular cleavage of the hFVIIa proteins occurs at Arg152, N-terminal sequencing of the heavy chain for each variant was performed. The observed amino acid sequence was identical to the expected IVGGK sequence in all variants, as was rhFVIIa (data not shown). Additionally, chemical $\gamma$-carboxyglutamic acid (Gla) analysis of the hFVII-WT, rFVIIa, and variants showed that $\gamma$-carboxylation was nearly complete (9 out of 10 expected Gla residues, data not shown). Of the three variants tested, hFVII-2RKR was most efficiently secreted in an activated form (Figure 2B), and it retains high activity (Figure 2C). In light of these results, we used this construct for subsequent in vivo experiments.

In vivo expression of $h F V I I-2 R K R$. A recombinant AAV2 vector with a liver-specific promoter/enhancer (hAAT promoter with apoE enhancer) driving expression of hFVII-2RKR (AAV2-hAAT-hFVII$2 \mathrm{RKR}$ ) was generated. Following its introduction into the hepatic circulation in immunodeficient C57BL/6 mice, we observed longterm and dose-dependent expression of $\mathrm{hFVII}-2 \mathrm{RKR}$ in the mouse circulation for up to 16 weeks (duration of experiment) (Figure 3). A dose range of $0.7 \times 10^{11}$ to $2 \times 10^{11}$ vector genomes $(\mathrm{vg})$ per mouse resulted in levels of approximately $150-250 \mathrm{ng} / \mathrm{ml}$, whereas a dose range of $0.6 \times 10^{12}$ to $1.2 \times 10^{12} \mathrm{vg} /$ mouse resulted in levels of approximately $650-980 \mathrm{ng} / \mathrm{ml}$, reaching a plateau of approximately $1 \mu \mathrm{g} / \mathrm{ml}$, as assayed by ELISA. Our results indicate that a minimum dose of $1.2 \times 10^{12} \mathrm{vg} /$ mouse was required to achieve circulating levels comparable to those shown to be clinically effective in humans $(2 \mu \mathrm{g} / \mathrm{ml})(6)$.

In vitro characterization of $m$ FVII and $m F V I I-2 R K R$. Previous work had shown that the complex of murine TF and hFVII has poor activity in a coagulation assay $(9,10)$, due to the low affinity of murine TF for hFVIIa (two orders of magnitude below that of human TF for hFVIIa (8). Therefore, expression of hFVIIa in the mouse circulation, though easily quantified with currently available $A b$ 's, may not effectively promote hemostasis if TF-dependent mechanisms account for the effect. To test the efficacy of our gene transfer approach in a more physiological system, we used the mouse homolog of our hFVII2RKR transgene. Using PCR, we cloned the mouse FVII cDNA (16) and engineered an HPC4 epitope tag at the $\mathrm{C}$ terminus of the protein. This allowed for rapid purification using affinity chromatography $(22,23)$. Under reducing conditions, mFVII-HPC4 was secreted as a single-chain molecule; in contrast, mFVII-2RKR-HPC4 was secreted as a two-chain molecule, with heavy and light chains of approximately 30 and $20 \mathrm{kDa}$, respectively (Figure 4A). A small percentage remained as a zymogen (Figure 4A). These results are similar to those obtained for the hFVII and hFVIIa variants (Figure 2B) and are in agreement with previously published data on the apparent molecular weight of $\mathrm{mFVII}$ (16). In vitro analysis of these proteins in a PT assay indicated that mFVII-2RKR-HPC4 was $63 \%$ as active as rhFVIIa (Figure 4B) and comparable to the hFVII-2RKR homolog (see Figure 2C). Further in vitro analyses in the presence of TF or FXa showed that both mFVII-HPC4 and mFVII-2RKR-HPC4 could be activated to near completion (data not shown), in agreement with our results using hFVII-WT and hFVII-2RKR. The data from the in vitro assays can be used to calculate specific activities of the murine and human variant VIIa proteins. Based on a specific activity of 50 kilo international units $/ \mathrm{mg}(\mathrm{KIU} / \mathrm{mg})$ protein for rhFVIIa (NovoSeven), cited in the manufacturer's product insert, the specific activity of hFVII-2RKR is $43 \mathrm{KIU} / \mathrm{mg}$, and that of mFVII2RKR-HPC4 is $32 \mathrm{KIU} / \mathrm{mg}$ protein.

In vivo expression of $m$ FVII-2RKR. To test the efficacy of the mFVII2RKR transgene, an AAV2-hAAT-mFVII-2RKR vector was introduced into the hepatic circulation of normal and hemophilia $\mathrm{B}$ C57BL/ 6 animals $\left(1.2 \times 10^{12} \mathrm{vg} / \mathrm{mouse}\right)$. We observed long-term reduction of both PT (Figure 5A) and aPTT (Figure 5B) in normal mice. Using an ELISA for $\mathrm{mFVII} / \mathrm{mFVII}-2 \mathrm{RKR}$ (based on a rabbit anti-mFVII/mFVIIa $\mathrm{Ab}$ that we generated for this study), we showed that the levels of total antigen were elevated about threefold above baseline, and a plateau was reached at about 1,200 $\mathrm{ng} / \mathrm{ml}$ (data not shown), in general agreement with our hFVII results at a comparable vector dose (see Figure 3). Delivery of the same vector and dose in immunocompetent hemophilia $\mathrm{B}$ mice led to sustained reduction in PT (Figure 5C), but, more importantly, the aPTT was lowered from levels typically seen in hemophilia B mice ( $\sim 65$ seconds) into the normal range ( $\sim 30$ seconds) (Figure $5 D)$. This level of correction has persisted unchanged for 22 weeks
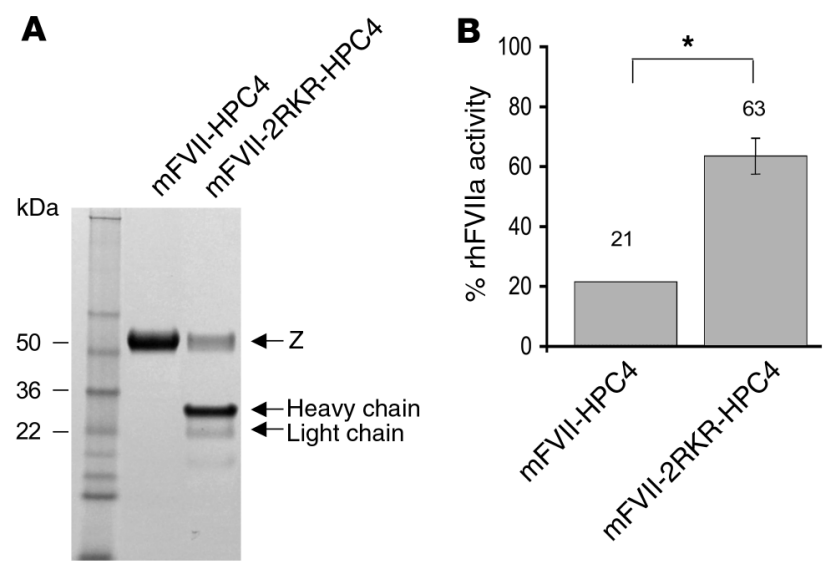

\section{Figure 4}

In vitro characterization and purification of $\mathrm{mFVII-HPC4}$ and $\mathrm{mFVII-}$ 2RKR-HPC4. (A) PAGE gel of $4 \mu \mathrm{g}$ of purified proteins, under reducing conditions. Numbers on the left side of the gel denote size in $\mathrm{kDa}$, whereas arrows on the right point to the zymogen form, heavy chain, and light chain. The light chain does not stain as intensely as the heavy chain, a previously noted finding (19). (B) In vitro activity based on PT as indicated on top of each bar (mean \%), relative to rhFVIla (100\%): left, $\mathrm{mFVII-HPC4}$, and right, mFVII-2RKR-HPC4. Error bars denote mean \pm 1 SD, ${ }^{*} P<0.001$. 

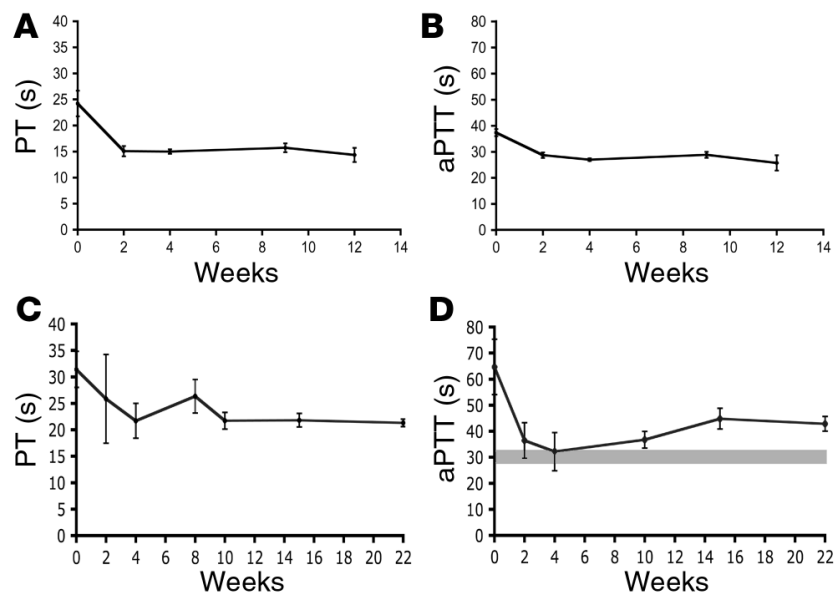

Figure 5

In vivo expression of mFVII-2RKR, as assayed by PT and aPTT, in immunocompetent, hemostatically normal $(n=4)$, and hemophilia B $(n=6)$ mice, following injection of $1.2 \times 10^{12} \mathrm{vg} /$ mouse. (A) PT and (B) aPTT in hemostatically normal C57BL/6 mice; (C) PT and (D) aPTT in hemophilia B mice on C57BL/6 background. The gray area in (D) represents the range of aPTT of normal mice.

in hemophilia B mice on a C57BL/6 background, with observation ongoing. Expression of functional mFVII-2RKR in hemophilia B animals was confirmed by measuring blood loss following a hemostatic challenge (tail clipping) (Figure 6). The tail was transected at a diameter of $3 \mathrm{~mm}$ in normal $(n=10)$, untreated hemophilia B $(n=10)$, and AAV-mFVII-2RKR-treated mice $(n=5)$, all on a C57BL/ 6 background, and blood loss was quantified by measuring the OD at $575 \mathrm{~nm}$ of the saline solution into which the tail was immersed for a 15-minute interval after transection. As shown in Figure 6, blood loss (as reflected by hemoglobin concentration) in the hemostatically normal mice is minimal, while in the hemophilia $B$ mice it is substantial $(P<0.0001$ between cohorts). In mice treated 6-8 weeks previously with AAV-mFVII-2RKR, blood loss was reduced to a statistically significant degree compared with hemophilia B mice $(P=0.001)$, although it was still significantly different from normal baseline $(P<0.0001)$. Thus AAV-mFVII-2RKR is able to effect hemostasis at the doses $\left(1.2 \times 10^{12} \mathrm{vg} /\right.$ mouse $)$ tested here.

As a surrogate measure of risk of thrombosis as a result of continuous expression of mVIIa, TAT levels were also measured. At the vector doses shown in Figure 5, levels of TAT were $36 \pm 4.6 \mu \mathrm{g} / \mathrm{l}$ before treatment (hemophilia B mice), and $44 \pm 12 \mu \mathrm{g} / \mathrm{l}$ at 8 weeks after AAV-mVII-2RKR injection ( $n=5$ mice). Thus there is no statistically significant change in TAT levels at this dose $(P=0.18)$. In addition, in a survival study (ongoing), $6 / 6$ hemophilia $B$ mice treated with AAV-mFVII-2RKR at $1.2 \times 10^{12} \mathrm{vg} / \mathrm{mouse}$ are alive and without evidence of thrombosis or other clinical sequelae 6 months after vector injection.

\section{Discussion}

The series of controlled proteolytic reactions that result in thrombin formation are divided into the extrinsic and intrinsic pathways (Figure 1). The X-linked bleeding diathesis that results from deficiencies of the intrinsic-pathway proteins hFVIII or hFIX is treated by intravenous infusion of the missing proteins, but a substantial fraction of patients develop inhibitory Ab's that block the activity of the infused proteins. One of the most important advances in hemophilia care in the last two decades has been the demonstration that clinical bleeding in such patients can be controlled by the infusion of rhFVIIa, the major enzyme in the extrinsic pathway. In fact, the hemostasis provided by rhFVIIa is so effective that for the first time patients with inhibitors have been able to undergo surgery without fear of life-threatening hemorrhagic complications (31). Based on the current understanding of the coagulation cascade, all patients with hemophilia could be treated with rhFVIIa, simplifying patient management to a single product, but the short half-life and high cost of rhFVIIa have prevented such an approach.

Gene transfer has been proposed as an alternative to protein infusion in the management of bleeding in hemophilia, but gene transfer also carries a risk of inhibitory $\mathrm{Ab}$ formation, as has been shown in hemophilic animal models with nearly every vector currently under investigation (18, 32-34). Gene transfer of FVIIa, however, circumvents the risk of inhibitor formation, since all hemophilia patients are tolerant to this endogenous protein. In addition, successful gene transfer obviates problems arising from a short halflife, since it confers long-term expression. The combination of gene transfer as the delivery method, and FVIIa as the transgene, thus offers a highly attractive novel approach to the problem of achieving hemostasis in hemophilia.

The rhFVIIa protein product currently in clinical use is produced as the single-chain form and activated to the two-chain form during protein purification. To effect a gene transfer approach, we needed to engineer a hFVII variant that would be secreted directly as hFVIIa. We inserted three potential recognition sequences for the intracellular protease PACE-furin and tested these for efficient, accurate removal of the inserted sequence and secretion of the twochain hFVIIa form. hFVII-2RKR proved best among the variants tested in terms of the low amounts of zymogen secreted and the near wild-type enzymatic activity (compared to rhFVIIa), and was thus used to prepare rAAV vectors.

Pharmacokinetic and clinical studies of rhFVIIa have established that circulating levels in the range of $2 \mu \mathrm{g} / \mathrm{ml}$ are required to achieve hemostasis in hemophilic patients with inhibitors. To determine the dose of vector (AAV-hAAT-hFVII-2RKR) needed to achieve appropriate circulating levels of hFVIIa, we began with a human transgene, since Ab's to hFVII/FVIIa are readily available and an

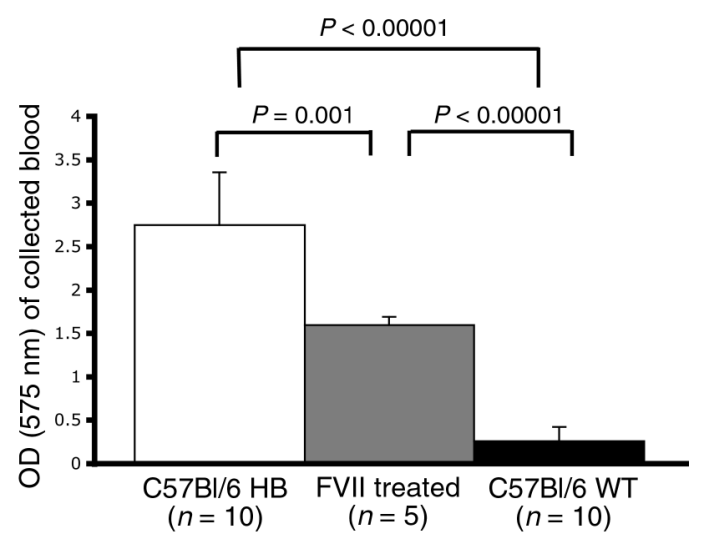

\section{Figure 6}

Blood loss measured by optical density after tail transection in AAV2-hAAT-mFVII-2RKR treated hemophilia B mice $(n=5)$, and in C57BL/6 normal $(n=10)$ and hemophilia B $(n=10)$ mice controls. $\mathrm{HB}$, hemophilia B. 
ELISA existed that allowed us to detect hFVIIa in murine plasma samples. As shown by our results, we achieved circulating hFVIIa levels of approximately $1 \mu \mathrm{g} / \mathrm{ml}$ using a dose of $1.2 \times 10^{12} \mathrm{vg} /$ mouse. Published literature documents a dose-response relationship in mice when rAAV is infused into the portal circulation (35) so achieving levels in the range of $2 \mu \mathrm{g} / \mathrm{ml}$ should not present difficulties, if indeed such levels are required (vide infra). Using these data as a guide, we switched to a vector encoding an engineered mFVIIa (mFVII-2RKR), since earlier studies had documented that, although mFVIIa forms an active complex with both human and murine TF, hFVIIa does not bind well to murine TF (8-10). Since mFVIIa binds well to human TF, we were able to use a standard PT assay, employing human TF (Innovin), to follow levels of mFVIIa activity in mouse plasma. The use of a murine transgene also allowed us to evaluate hemostasis in the hemophilic mouse model in response to a hemostatic challenge in vivo (tail clip assay). In the hemophilia mouse model, both the PT and aPTT shortened after steady-state levels of transgene expression were achieved, and the tail clip assay documented that the FVIIa levels achieved, approximately $1 \mu \mathrm{g} / \mathrm{ml}$, were adequate to reduce bleeding substantially in all treated hemophilia B mice compared with untreated animals. Moreover, at these levels of mFVIIa expression, there was no influence on survival out to 6 months (study still ongoing), and no biochemical evidence of increased risk of thrombosis in terms of elevated levels of thrombin-antithrombin compared with untreated hemophilia B controls. Additional studies at a range of doses and over prolonged periods will be required to define more clearly the risk of thrombosis in the face of continuous expression of FVIIa.

Although there is controversy in the literature about the precise mechanism by which FVIIa confers hemostasis, there is general consensus that a level of approximately $2 \mu \mathrm{g} / \mathrm{ml}$ is required to achieve hemostasis at least initially. Because of the way the protein product is used (repeated boluses with ensuing peak and trough levels), however, there are relatively few data on hemostatic (and potential adverse) effects of continuously maintained FVIIa levels. Nor is it clear whether continuous maintenance of some level of FVIIa lower than $2 \mu \mathrm{g} / \mathrm{ml}$ would allow spontaneous bleeds to be prevented. Certainly, in vitro model systems $(36,37)$ suggest that lower levels can improve the rate of thrombin generation compared with the untreated state. The modified FVIIa transgene that we describe here should allow us to test in animal models the hypothesis that lower levels of FVIIa, continuously expressed, are adequate to achieve hemostasis without promoting pathological thrombosis. Clinical experience with the protein product rFVIIa to date has revealed only a low level of thromboembolic phenomena, 17 episodes among 6,500 treated patients in one large series (38), but this experience may not be directly applicable to a gene transfer approach, where expression of FVIIa would be continuous rather than episodic. Extensive experience with continuously infused FVIIa protein is not yet available. It should be noted, however, that application of a FVIIa-gene transfer approach to humans with hemophilia would likely require the use of a regulatable promoter, so that circulating levels of FVIIa could be downregulated if necessary. Molecular 'switches' that allow promoter activity to rise and fall in response to administration of an oral drug are under development $(39,40)$.

The classic approach to gene transfer for genetic disease has been to use a vector to supply a normal copy of the defective gene. This approach carries with it the risk inherent to all protein replacement therapies, that is, a harmful immune response to the protein (or transgene product), which is perceived as "foreign" by the host immune system. In this report, we have described an alternate treatment strategy, in which the transgene product, to which the organism is fully tolerant, remedies the disease through a pathway independent of the missing protein. The keys to success in this instance (in addition to the biology of the coagulation cascade, which affords two pathways for the activation of FX) were the modified FVIIa transgene that could be secreted as activated FVIIa, and the gene transfer approach itself, which allows continuous expression and thus circumvents the dosing problems associated with a protein with a short half-life. Rapid advances in systems biology and proteomics may increasingly enable gene transfer approaches in which disease symptomatology is improved through administration of transgenes other than those affected by the mutation.

\section{Acknowledgments}

We acknowledge helpful discussions with Eleanor Pollak, Raffaella Toso, and Sriram Krishnaswamy. This work was supported by NIH grant NHLBI-U01-HL66948 to K.A. High, NIH grant NIDDK-K01060580 to V.R. Arruda, and by research grants from The Children's Hospital of Philadelphia and Hemophilia of Georgia.

Received for publication September 23, 2003, and accepted in revised form January 20, 2004.

Address correspondence to: Katherine A. High, 3615 Civic Center Boulevard, 302 Abramson Research Center, Philadelphia, Pennsylvania 19104, USA. Phone: (215) 590-4521; Fax: (215) 590-3660; E-mail: high@email.chop.edu.
1. Astermark, J., Ekman, M., and Berntorp, E. 2002. Antibodies to factor VIIa in patients with haemophilia and high-responding inhibitors. Br. J. Haematol. 119:342-347.

2. Hoyer, L.W. 1995. Why do so many haemophilia A patients develop an inhibitor? Br. J. Haematol. 90:498-501.

3. Shapiro, A. 2001. Inhibitor treatment: state of the art. Semin. Hematol. 38:26-34.

4. Lusher, J.M., et al. 1983. Autoplex versus proplex: a controlled, double-blind study of effectiveness in acute hemarthroses in hemophiliacs with inhibitors to Factor VIII. Blood. 62:1135-1138.

5. Sjamsoedin, L.J., et al. 1981. The effect of activated prothrombin-complex concentrate (FEIBA) on joint and muscle bleeding in patients with hemophilia A and antibodies to Factor VIII. A double-blind clinical trial. N. Engl. J, Med. 305:717-721.

6. Hedner, U. 2001. Recombinant Factor VIIa (Novoseven) as a hemostatic agent. Semin. Hematol.
38:43-47.

7. Lindley, C.M., et al. 1994. Pharmacokinetics and pharmacodynamics of recombinant Factor VIIa. Clin. Pharmacol. Ther. 55:638-648.

8. Nelsestuen, G.L., et al. 2001. Elevated function of blood clotting Factor VIIa mutants that have enhanced affinity for membranes. Behavior in a diffusion-limited reaction. J. Biol. Chem. 276:39825-39831.

9. Kadish, J.L., Wenc, K.M., and Dvorak, H.F. 1983. Tissue factor activity of normal and neoplastic cells: quantitation and species specificity. J. Natl. Cancer Inst. 70:551-557.

10. Janson, T.L., Stormorken, H., and Prydz, H. 1984. Species specificity of tissue thromboplastin. Haemostasis. 14:440-444.

11. Chuansumrit, A., Sangkapreecha, C., and Hathirat, P. 1999. Successful epistaxis control in a patient with Glanzmann thrombasthenia by increased bolus injection dose of recombinant factor VIIa.
Thromb. Haemost. 82:1778.

12. Mariani, G., Testa, M.G., Di Paolantonio, T., Molskov Bech, R., and Hedner, U. 1999. Use of recombinant, activated factor VII in the treatment of congenital factor VII deficiencies. Vox. Sang. 77:131-136.

13. Poon, M.C., et al. 2001. Use of recombinant factor VIIa (NovoSeven) in patients with Glanzmann thrombasthenia. Semin. Hematol. 38:21-25.

14. Peters, M., and Heijboer, H. 1998. Treatment of a patient with Bernard-Soulier syndrome and recurrent nosebleeds with recombinant factor VIIa. Thromb. Haemost. 80:352.

15. Pinotti, M., et al. 1998. Molecular mechanisms of FVII deficiency: expression of mutations clustered in the IVS7 donor splice site of factor VII gene. Blood. 92:1646-1651.

16. Idusogie, E., et al. 1996. Characterization of a cDNA encoding murine coagulation factor VII. Thromb. Haemost. 75:481-487. 
17. Okuyama, T., et al. 1996. Liver-directed gene therapy: a retroviral vector with a complete LTR and the ApoE enhancer-alpha 1-antitrypsin promoter dramatically increases expression of human alpha 1-antitrypsin in vivo. Hum. Gene Ther. 7:637-645.

18. Herzog, R.W., et al. 1999. Long-term correction of canine hemophilia B by gene transfer of blood coagulation factor IX mediated by adeno-associated viral vector. Nat. Med. 5:56-63.

19. Toso, R., Pinotti, M., High, K.A., Pollak, E.S., and Bernardi, F. 2002. A frequent human coagulation Factor VII mutation (A294V, c152) in loop 140s affects the interaction with activators, tissue factor and substrates. Biochem. J. 363:411-416.

20. Chu, G., and Sharp, P.A. 1981. SV40 DNA transfection of cells in suspension: analysis of efficiency of transcription and translation of T-antigen. Gene. 13:197-202.

21. Matsushita, T., et al. 1998. Adeno-associated virus vectors can be efficiently produced without helper virus. Gene Ther. 5:938-945.

22. Stearns, D.J., Kurosawa, S., Sims, P.J., Esmon, N.L., and Esmon, C.T. 1988. The interaction of $\mathrm{a} \mathrm{Ca}^{2+}$ dependent monoclonal antibody with the protein $\mathrm{C}$ activation peptide region. Evidence for obligatory $\mathrm{Ca}^{2+}$ binding to both antigen and antibody. J. Biol. Chem. 263:826-832.

23. Rezaie, A.R., and Esmon, C.T. 1992. The function of calcium in protein $\mathrm{C}$ activation by thrombin and the thrombin-thrombomodulin complex can be distinguished by mutational analysis of protein C derivatives. J. Biol. Chem. 267:26104-26109.
24. Ravanat, C., et al. 1995. Cross-reactivity of human molecular markers for detection of prethrombotic states in various animal species. Blood Coagul. Fibrinolysis. 6:446-455.

25. Lin, H.-F., Maeda, F., Smithies, O., Straight, D.L., and Stafford, D.W. 1997. A coagulation factor IXdeficient mouse model for hemophilia B. Blood. 90:3962-3966.

26. McVey, J.H., et al. 1998. Exclusion of the first EGF domain of factor VII by a splice site mutation causes lethal factor VII deficiency. Blood. 92:920-926.

27. Sambrano, G.R., Weiss, E.J., Zheng, Y.W., Huang, W., and Coughlin, S.R. 2001. Role of thrombin signalling in platelets in haemostasis and thrombosis. Nature. 413:74-78.

28. Wildgoose, P., et al. 1992. Measurement of basal levels of factor VIIa in hemophilia A and B patients [see comments]. Blood. 80:25-28.

29. van 't Veer, C., Golden, N.J., and Mann, K.G. 2000. Inhibition of thrombin generation by the zymogen factor VII: implications for the treatment of hemophilia A by factor VIIa. Blood. 95:1330-1335.

30. Golino, P. 2002. The inhibitors of the tissue factor:factor VII pathway. Thromb. Res. 106:V257-V265.

31. Shapiro, A.D., Gilchrist, G.S., Hoots, W.K., Cooper, H.A., and Gastineau, D.A. 1998. Prospective, randomised trial of two doses of rFVIIa (NovoSeven) in haemophilia patients with inhibitors undergoing surgery. Thromb. Haemost. 80:773-778.

32. Herzog, R.W., et al. 2002. Influence of vector dose on factor IX-specific T and B cell responses in muscledirected gene therapy. Hum. Gene Ther. 13:1281-1291.
33. Mount, J.D., et al. 2002. Sustained phenotypic correction of hemophilia B dogs with a factor IX null mutation by liver-directed gene therapy. Blood. 99:2670-2676.

34. Gallo-Penn, A.M., et al. 2001. Systemic delivery of an adenoviral vector encoding canine factor VIII results in short-term phenotypic correction, inhibitor development, and biphasic liver toxicity in hemophilia A dogs. Blood. 86:107-113.

35. Grimm, D., et al. 2003. Preclinical in vivo evaluation of pseudotyped adeno-associated virus vectors for liver gene therapy. Blood. 102:2412-2419.

36. Butenas, S., Brummel, K.E., Branda, R.F., Paradis, S.G., and Mann, K.G. 2002. Mechanism of factor VIIa-dependent coagulation in hemophilia blood. Blood. 99:923-930.

37. Turecek, P.L., et al. 2003. Factor VIII inhibitorbypassing agents act by inducing thrombin generation and can be monitored by a thrombin generation assay. Pathophysiol. Haemost. Thromb. 33:16-22.

38. Roberts, H.R. 2001. Recombinant factor VIIa (Novoseven) and the safety of treatment. Semin. Hematol. 38:48-50.

39. Chong, H., Ruchatz, A., Clackson, T., Rivera, V.M., and Vile, R.G. 2002. A system for small-molecule control of conditionally replication-competent adenoviral vectors. Mol. Ther. 5:195-203.

40. Lamartina, S., et al. 2003. Construction of an rtTA2(s)-m2/tts(kid)-Based transcription regulatory switch that displays no basal activity, good inducibility, and high responsiveness to doxycycline in mice an non-human primates. Mol. Ther. 7:271-280. 